\title{
Regularization and Extrapolation Methods for Infrared Divergent Loop Integrals
}

\author{
Elise de Doncker ${ }^{1}$, Shujun $\mathrm{Li}^{1}$, Yoshimitsu Shimizu ${ }^{2}$, \\ Junpei Fujimoto ${ }^{2}$, and Fukuko Yuasa ${ }^{2}$ \\ 1 Western Michigan University, \\ \{elise, sli\}@cs.wmich.edu \\ http://www.cs.wmich.edu/ ${ }^{\text {elise }}$ \\ 2 High Energy Accelerator Research Organization(KEK), \\ Oho 1-1, Tsukuba, Ibaraki, 305, Japan \\ \{yoshimitsu.shimizu, junpei.fujimoto, fukuko.yuasa\}@kek.jp
}

\begin{abstract}
Loop integrals occur in higher order perturbation calculations for the cross section of particle interactions in high energy physics. In previous work we introduced a numerical extrapolation method to handle a class of Feynman loop diagrams where the integrand shows a singular behavior on a hypersurface which may intersect the domain of integration. The integral is considered in the limit as a parameter in the integrand tends to zero. Under certain conditions, the extrapolation process achieves convergence acceleration to the limit. In order to handle massless cases, we apply a dimensional regularization technique to extract infrared divergences from the integral. We illustrate the combined technique using a scalar one-loop sample integral.
\end{abstract}

\section{Introduction}

Loop integrals occur in higher order perturbation terms of the scattering amplitude, which is used for cross section computations of particle interactions in high energy physics. The cross section of a particle interaction gives the probability of a given configuration in energy-momentum space $\left(E, p^{1}, p^{2}, p^{3}\right)$.

Figure 1 gives an example of a one-loop Feynman diagram [2. In a Feynman diagram, each line, termed propagator is associated with a particle and can be straight or wavy depending on whether the particle is a fermion or boson, respectively. $M$ and $m$ are particle masses. A propagator corresponds to an intermediate state of a particle, where it is not observable. Particles collide at the vertices of the diagram, according to a coupling constant $g$ which represents the strength of the interaction.

The diagram of Figure 1 is a one-loop diagram as it exhibits a single loop. No-loop (tree diagram) and multi-loop configurations are possible. The number of vertices $N$ specifies the type of the diagram (e.g., vertex for $N=3$ and box for $N=4)$.

The scattering amplitude $T$ is expanded as a (perturbation) series in $g$,

$$
T=T_{0}+T_{1} g+T_{2} g^{2}+\ldots
$$




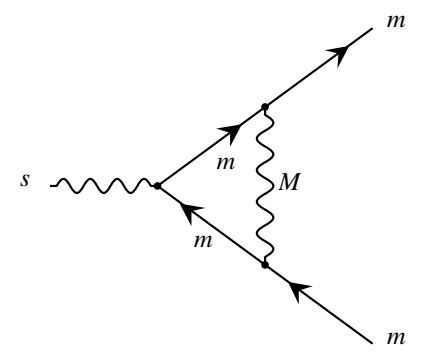

produced by GRACEFIG

Fig. 1. Vertex example

Here, $T_{0}$ represents the tree amplitude, and $T_{k}$ the $k$-loop amplitude for $k \geq 1$. The cross section is then derived as a series from (11) (see 8]).

A general form of the scalar one-loop $N$-point integral is given in 3 , as a 4-dimensional integral over the loop momenta. In [8], a sample one-loop integral with three propagators is introduced as

$$
\mathcal{I}=\frac{1}{(2 \pi)^{4} i} \int_{-\infty}^{\infty} \mathrm{d}^{4} l \frac{1}{\left((p-l)^{2}-m^{2}+i \varepsilon\right)\left((q+l)^{2}-m^{2}+i \varepsilon\right)\left(\left(l^{2}-M^{2}+i \varepsilon\right)\right.},
$$

where $l$ is the loop momentum, and $p$ and $q$ are given momenta which are assumed to satisfy $p^{2}=q^{2}=m^{2}$. Furthermore, $\varepsilon>0$ is a real constant which is supplied to prevent the integral from diverging. The physical scattering amplitude contains this type of integrals and its value is defined at $\varepsilon=0$. Using the identity

$$
\frac{1}{A_{1} A_{2} A_{3}}=\Gamma(3) \int_{0}^{1} \mathrm{~d} x_{1} \int_{0}^{1-x_{1}} \mathrm{~d} x_{2} \frac{1}{\left(x_{1} A_{1}+x_{2} A_{2}+\left(1-x_{1}-x_{2}\right) A_{3}\right)^{3}}
$$

and integrating over $l$ then results in

$$
\mathcal{I}=-\frac{1}{16 \pi^{2}} \int_{0}^{1} \mathrm{~d} x_{1} \int_{0}^{1-x_{1}} \mathrm{~d} x_{2} \frac{1}{\mathcal{F}\left(x_{1}, x_{2}\right)-i \varepsilon}
$$

with

$$
\mathcal{F}\left(x_{1}, x_{2}\right)=-s x_{1} x_{2}+m^{2}\left(x_{1}+x_{2}\right)^{2}+M^{2}\left(1-x_{1}-x_{2}\right)
$$

$\left(s=(p+q)^{2}\right.$ denotes the squared energy). The integral $\mathcal{I}$ of (2) is called a scalar loop integral as the numerator of the integrand equals 1 . In a non-scalar integral, the numerator is generally a polynomial.

A generalization to $N$ particles can be written as

$$
I=\int_{0}^{\infty} \mathrm{d} x_{1} \mathrm{~d} x_{2} \ldots \mathrm{d} x_{N} \frac{\delta\left(1-\sum_{j=1}^{N} x_{j}\right)}{\left(\left(\mathcal{F}_{N}\left(x_{1}, x_{2}, \ldots, x_{n}\right)-i \varepsilon\right)^{N-2}\right.} .
$$


While analytical formulations were given for scalar one-loop integrals, e.g., in [9, 10, there is considerable interest in numerical methods which could handle non-scalar cases in a similar way.

In [2] we introduced a numerical extrapolation method to handle Feynman loop diagrams where the integrand shows a singular behavior on a hypersurface which may intersect the domain of integration. The scalar 1-loop vertex integral was considered as a study case.

The extrapolation procedure yields an approximation to the limit as $\varepsilon$ tends to 0 , under certain conditions on the asymptotic behavior of the integral with respect to $\varepsilon$. The infrared singularity, where $M / m \rightarrow 0$, affects the asymptotic behavior. The effect of the singularity (at $x_{1}=x_{2}=0$ ) emerges in the integral (2) above as $M$ is set to 0 in (3).

In this paper we investigate a combination of the extrapolation for $\varepsilon \rightarrow 0$ with the dimensional regularization method by Binoth and Heinrich [1]. The latter extracts poles introduced by the infrared singularity (but does not handle the internal singularity). Dimensional regularization is applied in QCD (Quantum Chromodynamics) which deals with the strong interactions between quarks and gluons (both are massless).

\section{Regularization Techniques}

One method to regulate the infrared divergence is by using the fictitious mass $\lambda$ of the photon as $M=\lambda$. This results in a singular behavior of the form $\log \lambda$ when (2) is integrated analytically.

Another method uses dimensional regularization. For the latter, we re-write the exponent of the denominator in (4) as $N-D / 2$ with the dimension $D=4-2 \eta$ as $\eta \rightarrow 0$. As shown in [1, this results in a Laurent expansion of the integral as a function of $\eta$, from which the poles can be extracted.

Both methods introduce non-physical situations: the mass of the photon does not correspond to nature, and the extra dimension from 4 is also non-physical. This implies that the final results should not depend on the fictitious photon mass, or on $1 / \eta$. The Kinoshita-Lee-Nauenberg theorem [4, 5, 6] implies that, after accumulating the amplitude with respect to one-loop diagrams and emission of the photon, the infrared divergence disappears, i.e., the terms involving the photon mass vanish; or the coefficient of the $1 / \eta$ term resulting in the dimensional regularization is zero.

The (linear) extrapolation procedure of 2 gives good extrapolated results for $m=m_{e}=0.51110^{-3} \mathrm{GeV}, M=10^{-5} \mathrm{GeV}$ and $s=100 \mathrm{GeV}^{2}$, using small $\varepsilon$ values in the integrand. As an example, the approximated imaginary part gives 0.86805413, in agreement with the analytical expression given in [3], for $\varepsilon=2^{-32}\left(* 2^{-1}\right) 2^{-41}$ and double precision arithmetic. This run takes time of the order of 20-25 seconds on a $2.5 \mathrm{GHz}$ Athlon processor laptop.

The convergence behavior is illustrated for the real part in Table 1. For the $\varepsilon$ values listed in the leftmost column, the numerical integration results for (2) (disregarding the constant factor $-1 /\left(16 \pi^{2}\right)$ ) are listed in column 2, 
Table 1. Extrapolation Results for $m=m_{e}=0.51110^{-3} \mathrm{GeV}, M=10^{-5} \mathrm{GeV}$ and $s=100 \mathrm{GeV}^{2}$

\begin{tabular}{|l|ccr|}
\hline$\varepsilon$ & $\begin{array}{c}\text { Integration } \\
\text { result }\end{array}$ & $\begin{array}{c}\text { Extrapolated } \\
\text { result }\end{array}$ & $\begin{array}{r}\text { \#Function } \\
\text { evals. }\end{array}$ \\
\hline $2^{-32}$ & $-0.3295240321026314 \mathrm{E}+01$ & & \\
$2^{-33}$ & $-0.3384924822405812 \mathrm{E}+01$ & $-0.3474609323785311 \mathrm{E}+01$ & $0.28055550 \mathrm{E}+07$ \\
$2^{-34}$ & $-0.3430352719735732 \mathrm{E}+01$ & $-0.3476171048159099 \mathrm{E}+01$ & $0.30822750 \mathrm{E}+07$ \\
$2^{-35}$ & $-0.3443533393938414 \mathrm{E}+01$ & $-0.3446671052357742 \mathrm{E}+01$ & $0.34816650 \mathrm{E}+07$ \\
$2^{-36}$ & $-0.3445153187362171 \mathrm{E}+01$ & $-0.3442193850326485 \mathrm{E}+01$ & $0.38417550 \mathrm{E}+07$ \\
$2^{-37}$ & $-0.3444447779180230 \mathrm{E}+01$ & $-0.3442652948552781 \mathrm{E}+01$ & $0.42755850 \mathrm{E}+07$ \\
$2^{-38}$ & $-0.3443700367400504 \mathrm{E}+01$ & $-0.3442689614444009 \mathrm{E}+01$ & $0.46413150 \mathrm{E}+07$ \\
$2^{-39}$ & $-0.3443227592907696 \mathrm{E}+01$ & $-0.3442688989410037 \mathrm{E}+01$ & $0.50845950 \mathrm{E}+07$ \\
$2^{-40}$ & $-0.3442966496374880 \mathrm{E}+01$ & $-0.3442688970861624 \mathrm{E}+01$ & $0.56085150 \mathrm{E}+07$ \\
$2^{-41}$ & $-0.3442829784757492 \mathrm{E}+01$ & $-0.3442688970926735 \mathrm{E}+01$ & $0.60628650 \mathrm{E}+07$ \\
$2^{-42}$ & $-0.3442759890277537 \mathrm{E}+01$ & $-0.3442688970927178 \mathrm{E}+01$ & $0.66527850 \mathrm{E}+07$ \\
$2^{-43}$ & $-0.3442724558666787 \mathrm{E}+01$ & $-0.3442688970927342 \mathrm{E}+01$ & $0.74449050 \mathrm{E}+07$ \\
\hline
\end{tabular}

and the corresponding extrapolated results using linear extrapolation in column 3. The integration was performed iteratively using a one-dimensional code in each direction (DQAGE from Quadpack [7]), with a relative error tolerance of $10^{-12}$.

For $M=10^{-5}$ or $10^{-7} \mathrm{GeV}$ and $s=100,10,000$ or $100,000 \mathrm{GeV}^{2}$, results can be obtained to 5 or 6 figure accuracy (starting from, e.g., $\varepsilon=2^{-42}$, and for a requested integration accuracy of $10^{-7}$ or $10^{-8}$ for the integral approximations corresponding to each of the $\varepsilon$ values).

It is interesting to note that, even though the procedure breaks down for $M=10^{-9} \mathrm{GeV}$ using double precision arithmetic, we are able to extend it to $M=10^{-15} \mathrm{GeV}$ using quadruple precision and a total number of function evaluations of the order of $10^{7}$ for a requested relative accuracy of $10^{-7}$. The method works in cases where the integrals can be obtained efficiently for very small $\varepsilon$ values.

\section{Dimensional Regularization}

In this section we apply a dimensional regularization technique. According to 1 we split the integral (2) into sector integrals. We will omit the term $-i \varepsilon$ in the notation below initially; it is re-introduced later for the computation.

We start from the form

$$
I=\int_{0}^{\infty} d x_{1} \int_{0}^{\infty} d x_{2} \int_{0}^{\infty} d x_{3} \frac{\delta\left(1-x_{1}-x_{2}-x_{3}\right)}{\left(-s x_{1} x_{2}+m^{2}\left(x_{1}+x_{2}\right)^{2}+M^{2} x_{3}\right)^{N-D / 2}}
$$

of dimension $N=3$ and where $D=4-2 \eta$. As we are interested in the infrared singularity, we will consider the case where $M \rightarrow 0$ (and $m$ is fixed). 
The domain is split into $N$ sectors, which in this case are given by:

$$
\begin{aligned}
& \left\{\left(x_{1}, x_{2}, x_{3}\right) \mid 0 \leq x_{2} \leq x_{1}, 0 \leq x_{3} \leq x_{1}\right\}, \\
& \left\{\left(x_{1}, x_{2}, x_{3}\right) \mid 0 \leq x_{1} \leq x_{2}, 0 \leq x_{3} \leq x_{2}\right\}, \\
& \left\{\left(x_{1}, x_{2}, x_{3}\right) \mid 0 \leq x_{1} \leq x_{3}, 0 \leq x_{2} \leq x_{3}\right\} .
\end{aligned}
$$

The integral $I_{1}$ over the first sector is thus as $I$ but where the integration limits of the $x_{2}$ and $x_{3}$ ranges are replaced by $\left(0, x_{1}\right)$. Performing the transformation

$$
\begin{aligned}
& x_{1} \\
& x_{2}=x_{1} t_{1} \\
& x_{3}=x_{1} t_{2}
\end{aligned}
$$

in (5) yields

$$
I_{1}=\int_{0}^{\infty} d x_{1} \int_{0}^{1} d t_{1} \int_{0}^{1} d t_{2} \frac{\delta\left(1-x_{1}\left(1+t_{1}+t_{2}\right)\right)}{x_{1}^{2 N-D-2}\left(-s t_{1}+\left(1+t_{1}\right)^{2} m^{2}+M^{2} \frac{t_{2}}{x_{1}}\right)^{N-D / 2}} .
$$

We now use the transformation $x_{1}=y_{1} /\left(1+t_{1}+t_{2}\right)$, so that

$$
I_{1}=\int_{0}^{\infty} d y_{1} \int_{0}^{1} d t_{1} \int_{0}^{1} d t_{2} \frac{\delta\left(1-y_{1}\right)\left(1+t_{1}+t_{2}\right)^{2 N-D-3}}{y_{1}^{2 N-D-2}\left(-s t_{1}+\left(1+t_{1}\right)^{2} m^{2}+M^{2} \frac{t_{2}\left(1+t_{1}+t_{2}\right)}{y_{1}}\right)^{N-D / 2}},
$$

and $y_{1}$ integrates out in view of the delta function, giving

$$
I_{1}=\int_{0}^{1} d t_{1} \int_{0}^{1} d t_{2} \frac{\left(1+t_{1}+t_{2}\right)^{2 N-D-3}}{\left(-s t_{1}+\left(1+t_{1}\right)^{2} m^{2}+M^{2} t_{2}\left(1+t_{1}+t_{2}\right)\right)^{N-D / 2}} .
$$

The integral $I_{2}$ over the second sector equals $I_{1}$ through symmetry. Similar operations on the third sector integral cast it into the form

$$
I_{3}=\int_{0}^{1} d t_{1} \int_{0}^{1} d t_{2} \frac{\left(1+t_{1}+t_{2}\right)^{2 N-D-3}}{\left(-s t_{1} t_{2}+\left(t_{1}+t_{2}\right)^{2} m^{2}+M^{2}\left(1+t_{1}+t_{2}\right)\right)^{N-D / 2}} .
$$

The extrapolation procedure of [2] applied to the sum of $I_{1}, I_{2}$ and $I_{3}$ gives the same results as when applied to $I$, for example, with $m=40 \mathrm{GeV}, M=93 \mathrm{GeV}$, $s=9000 \mathrm{GeV}^{2}$ and $\varepsilon$ (for the extrapolation) ranging over $128\left(* 2^{-1}\right) 1$.

\section{$4 \quad$ Infrared Singularity}

Examination of the sector integrals obtained previously as $M \rightarrow 0$ indicates that only $I_{3}$ in (7) shows the infrared singularity; indeed its denominator

$$
\left(-t_{1} t_{2} s+\left(t_{1}+t_{2}\right)^{2} m^{2}\right)^{N-D / 2} \rightarrow 0
$$

as both $t_{1}$ and $t_{2}$ tend to zero, and $N-D / 2=N-2+\eta=1+\eta \rightarrow 1$ as $\eta \rightarrow 0$. 
We write $I_{3}=I_{3}^{\prime}+I_{3}^{\prime \prime}$ where

$$
I_{3}^{\prime}=\int_{0}^{1} d t_{1} \int_{0}^{t_{1}} d t_{2} \frac{\left(1+t_{1}+t_{2}\right)^{2 N-D-3}}{\left(-s t_{1} t_{2}+\left(t_{1}+t_{2}\right)^{2} m^{2}+M^{2}\left(1+t_{1}+t_{2}\right)\right)^{N-D / 2}}
$$

and

$$
I_{3}^{\prime \prime}=\int_{0}^{1} d t_{2} \int_{0}^{t_{2}} d t_{1} \frac{\left(1+t_{1}+t_{2}\right)^{2 N-D-3}}{\left(-s t_{1} t_{2}+\left(t_{1}+t_{2}\right)^{2} m^{2}+M^{2}\left(1+t_{1}+t_{2}\right)\right)^{N-D / 2}}
$$

(note that $I_{3}^{\prime}=I_{3}^{\prime \prime}$ through symmetry). Performing the transformation $t_{1}=t_{2} t_{1}^{\prime}$ in $I_{3}^{\prime \prime}$ and setting $M=0$ gives

$$
I_{3}^{\prime \prime}=\int_{0}^{1} d t_{2} \int_{0}^{1} d t_{1}^{\prime} \frac{t_{2}^{D+1-2 N}\left(1+t_{2} t_{1}^{\prime}+t_{2}\right)^{2 N-D-3}}{\left(-s t_{1}^{\prime}+\left(t_{1}^{\prime}+1\right)^{2} m^{2}\right)^{N-D / 2}} .
$$

Let us write this as

$I_{3}^{\prime \prime}=\int_{0}^{1} d t_{1}^{\prime} \frac{1}{\left(-s t_{1}^{\prime}+\left(t_{1}^{\prime}+1\right)^{2} m^{2}\right)^{N-D / 2}} \int_{0}^{1} d t_{2} t_{2}^{D+1-2 N}\left(1+t_{2} t_{1}^{\prime}+t_{2}\right)^{2 N-D-3}$,

where the exponent of $t_{2}$ is $D+1-2 N=-1-2 \eta$, which tends to -1 as $\eta \rightarrow 0$. This corresponds to a logarithmic behavior caused by the infrared singularity.

With respect to the singularity at $t_{2}=0$, we expand the part

$$
f\left(t_{2}, \eta\right)=\left(1+t_{2} t_{1}^{\prime}+t_{2}\right)^{2 N-D-3}
$$

around $t_{2}=0$. Since we set $D=4-2 \eta$, the exponent of the singular factor in $t_{2}$ is $D+1-2 N=4-2 \eta+1-6=-1-2 \eta$. Thus we expand

$$
f\left(t_{2}, \eta\right)=f^{(0)}(0, \eta)+R\left(t_{2}, \eta\right)
$$

with the remainder term

$$
R\left(t_{2}, \eta\right)=f\left(t_{2}, \eta\right)-f^{(0)}(0, \eta)=f\left(t_{2}, \eta\right)-1 .
$$

Substituting for $f\left(t_{2}, \eta\right)$ in $I_{3}^{\prime \prime}$ gives

$$
I_{3}^{\prime \prime}=\int_{0}^{1} d t_{1}^{\prime} \frac{1}{\left(-s t_{1}^{\prime}+\left(t_{1}^{\prime}+1\right)^{2} m^{2}\right)^{N-D / 2}} \int_{0}^{1} d t_{2} t_{2}^{D+1-2 N}\left(f^{(0)}(0, \eta)+R\left(t_{2}, \eta\right)\right) .
$$

Thus, with respect to the infrared singularity, the integral is split into two terms, the first one of which accounts for the pole as $\eta \rightarrow 0$. The finite part of $I_{3}^{\prime \prime}$ is derived from the term in $R\left(t_{2}, \eta\right)$,

$$
\int_{0}^{1} d t_{1}^{\prime} \frac{1}{\left(-s t_{1}^{\prime}+\left(t_{1}^{\prime}+1\right)^{2} m^{2}\right)^{N-D / 2}} \int_{0}^{1} d t_{2} t_{2}^{D+1-2 N} R\left(t_{2}, \eta\right) .
$$

Numerical results can be obtained converging to the finite part by setting $\eta=0$ and introducing (extrapolating on) the $\varepsilon$ parameter to account for the singularity inside the integration region. 


\section{Conclusions}

The dimensional regularization technique of Binoth and Heinrich [1] leads to obtaining a Laurent series expansion as a function of $\eta$. Their method does not deal with integrand singularities inside the region of integration. To handle a singularity on a quadratic which intersects the integration region, we introduce a parameter $\varepsilon$ in the integrand and perform an extrapolation as $\varepsilon$ tends to 0 . This technique enables us to evaluate the finite part integral.

Furthermore we consider a regularization with respect to the photon mass parameter, results of which depend on the arithmetic precision used for the computation.

\section{References}

1. Binoth, T., And Heinrich, G. An automized algorithm to compute infrared divergent multi-loop integrals. hep-ph/0004013 v2.

2. De Doncker, E., Shimizu, Y., Fujimoto, J., And Yuasa, F. Computation of loop integrals using extrapolation. Computer Physics Communications 159 (2004), $145-156$.

3. Fujimoto, J., Shimizu, Y., Kato, K., and Oyanagi, Y. Numerical approach to one-loop integrals. Progress of Theoretical Physics 87, 5 (1992), 1233-1247.

4. Kinoshita, T. J. Math Phys. 3 (1962), 650.

5. Lee, T. D., And Nauenberg, M. Phys. Rev. 133 (1964), 1549.

6. Nakanishi, N. Prog. Theor. Phys. 19 (1958), 150.

7. Piessens, R., de Doncker, E., Überhuber, C. W., and Kahaner, D. K. QUADPACK, A Subroutine Package for Automatic Integration. Springer Series in Computational Mathematics. Springer-Verlag, 1983.

8. Shimizu, Y. Glossary for perturbative calculations in quantum field theory, August 2002.

9. 'т Hooft, G., And Veltman, M. Nucl. Phys. B 153 (1979), 365.

10. van Oldenborgh, G. J., And Vermaseren, J. A. M. Z. Phys. C 46 (1990), 425. 